\title{
INFLUÊNCIA DA RELAÇÃO CÁLCIO:MAGNÉSIO DO CORRETIVO NA NODULAÇÃO, PRODUÇÃO E COMPOSIÇÃO MINERAL DA ALFAFA ${ }^{1}$
}

\author{
ADÔNIS MOREIRA² ${ }^{2}$ JANICE GUEDES DE CARVALHO ${ }^{3}$ e ANTÔNIO RICARDO EVANGELISTA ${ }^{4}$
}

\begin{abstract}
RESUMO - O objetivo deste trabalho foi avaliar o efeito da relação Ca:Mg do corretivo da acidez do solo na produção de matéria seca, composição mineral da alfafa, e nodulação. O solo utilizado foi um Latossolo Vermelho-Escuro distrófico. Utilizou-se o delineamento inteiramente casualizado em esquema fatorial com seis relações de Ca: $\operatorname{Mg}\left(1: 0 ; 1: 1 ; 2: 1 ; 3: 1 ; 4: 1\right.$, na dosagem de 3,9 tha ${ }^{-1}$ do corretivo e uma relação 3:1 na dosagem de 7,8 $\mathrm{t} \mathrm{ha}^{-1}$ ) x seis épocas de corte, com quatro repetições. Mediram-se as produções de matéria seca (MS), teores de N, P, K, Ca, Mg e S na parte aérea, e peso fresco e massa seca dos nódulos, determinados no último corte. As mudanças na relação Ca:Mg não afetaram a produção de MS da alfafa. O dobro da dosagem de calcário recomendada apresentou a maior produção de MS. O teor de $\mathrm{Ca}$ e $\mathrm{Mg}$ foi diretamente proporcional à sua disponibilidade no solo, ao passo que o $\mathrm{K}$ foi inversamente proporcional ao teor de Ca. Não houve influência dos tratamentos nos teores de P. As relações $\mathrm{Ca}: \mathrm{Mg}$ afetaram os teores de $\mathrm{S}$ na matéria seca, os quais foram menores nos tratamentos com maior quantidade de $\mathrm{Ca}$. O tratamento 4:1 apresentou maior teor de $\mathrm{N}$, e as maiores concentração de Ca no solo aumentaram a fixação de $\mathrm{N}$ à planta.
\end{abstract}

Termos para indexação: cerrado, formação de nódulos, eficiência da nodulação, Latossolo VermelhoEscuro distrófico, matéria seca, Medicago sativa, nódulos, concentração de nutrientes.

\section{INFLUENCE OF CALCIUM:MAGNESIUM RATIO IN LIMESTONE ON NODULATION, DRY MATTER YIELD AND MINERAL COMPOSITION OF ALFALFA}

\begin{abstract}
This study evaluated the effect of different Ca:Mg ratios on dry matter yield, mineral composition of alfalfa, and nodulation. A dystrophic Dark-Red Latosol (Oxisol) soil was used in the experiment. A randomized design was adapted with five Ca:Mg ratios $(1: 0 ; 1: 1 ; 2: 1 ; 3: 1$ and $4: 1)$ at a recommended limestone dosage of $3.9 \mathrm{t} \mathrm{ha}^{-1}$. An additional treatment was included at a ratio of $3: 1$ with the dosage of $7.8 \mathrm{t} \mathrm{ha}^{-1}$. All treatments had four replicates in six cutting times. The analyzed variables were dry matter production, concentrations $\left(\mathrm{g} \mathrm{kg}^{-1}\right)$ of $\mathrm{N}, \mathrm{P}, \mathrm{K}, \mathrm{Ca}, \mathrm{Mg}$ and $\mathrm{S}$ in dry matter and weights of fresh and dry nodules. The changes in the ratios of $\mathrm{Ca}: \mathrm{Mg}$ at the recommended limestone rate did not affect the dry matter production. The highest dry matter yield was obtained in the treatment that used twice the recommended dosage. The concentrations of $\mathrm{Ca}$ and $\mathrm{Mg}$ in the aerial dry matter were directly proportional to their availability in the soil, while the $\mathrm{K}$ concentrations were inversely proportional to the rate $\mathrm{Ca}$ applied. No treatment influenced the concentration of $\mathrm{P}$ in the aerial dry matter. The concentration of $\mathrm{S}$ was influenced by the ratio of $\mathrm{Ca}: \mathrm{Mg}$ and diminished with increases in $\mathrm{Ca}$. The $4: 1$ treatment showed the highest concentration of $\mathrm{N}$ in the dry matter. The $\mathrm{N}$ fixation enhanced as the $\mathrm{Ca}$ rate in the soil increased.

Index terms: cerrado, nodule formation, nodule efficiency, dystrophic Dark-Red Latosol, Oxisol, dry matter, Medicago sativa, nodules, nutrient concentration.
\end{abstract}

${ }^{1}$ Aceito para publicação em 30 de junho de 1998.

Financiado pela FAPEMIG.

${ }^{2}$ Eng. Agr., M.Sc., Doutorando no CENA/USP, Caixa Postal 96, CEP 13400-970 Piracicaba, SP. Bolsista da FAPESP. E-mail: amoreira@pira.cena.usp.br

${ }^{3}$ Eng ${ }^{\mathrm{a}}$ Agr ${ }^{\mathrm{a}}$, Dr ${ }^{\mathrm{a}}$, Prof ${ }^{\mathrm{a}}$ Titular, Dep. de Ciência do Solo, Universidade Federal de Lavras (UFLA), Caixa Postal 37, CEP 37200-000 Lavras, MG. Bolsista do CNPq.

${ }^{4}$ Eng. Agr., D.Sc., Prof. Titular, Dep. de Zootecnia, UFLA. Bolsista do CNPq.

\section{INTRODUÇÃO}

A alfafa (Medicago sativa L.) é uma planta forrageira perene, originária do sudoeste da Ásia, de onde se difundiu para a Europa e para as Américas. No Brasil, a sua entrada deve ter sido pelo Rio Grande do Sul, através do Uruguai e Argentina. Existem também evidências de que os imigrantes italianos e 
alemães tenham trazido as sementes diretamente da Europa, cultivando-as nos vales dos rios e regiões coloniais, nos quais a fertilidade natural dos solos permitiu sua expansão; daí foi difundida para Santa Catarina, Paraná (Nuernberg, 1986) e Minas Gerais, que têm área cultivada de aproximadamente 3.000 ha com produção média de $8 \mathrm{t} \mathrm{ha}^{-1}$ de feno por ano (Anuário Estatístico do Brasil, 1989).

O interesse no cultivo da alfafa está relacionado principalmente às suas qualidades nutritivas excepcionais. É rica em proteínas, cálcio, fósforo e vitaminas $\mathrm{A}, \mathrm{B}_{1}, \mathrm{~B}_{2} \mathrm{C}$, E e $\mathrm{K}$, produzindo forragem tenra, suculenta e muito palatável (Nuernberg, 1986). Por ser uma planta rica em minerais, a alfafa exige boas condições de fertilidade do solo, tanto em relação ao $\mathrm{pH}$ quanto na disponibilidade de nutrientes (Nuernberg et al., 1990). Para que isso ocorra, é necessário corrigir a acidez do solo, o que pode ser feito utilizando-se unicamente carbonato de cálcio como elemento de correção da acidez e neutralização do alumínio trocável (Tisdale et al., 1993).

A calagem provoca um aumento no número de nódulos, na fixação de $\mathrm{N}$ e no acúmulo de matéria seca (Silva, 1981). Tais efeitos são atribuídos ao aumento na disponibilidade de nutrientes como $\mathrm{P}, \mathrm{Ca}$, $\mathrm{Mg}$, Mo e diminuição do $\mathrm{Al}$ e Mn trocáveis no solo. Por outro lado, a calagem excessiva pode acentuar uma eventual deficiência de K e micronutrientes, com exceção do Mo (Malavolta, 1980). Além disso, esse procedimento pode causar um desequilíbrio entre $\mathrm{Ca}$ e $\mathrm{Mg}$, prejudicando o crescimento das plantas (Nuernberg et al., 1990).

O suprimento de Ca e Mg está normalmente vinculado à aplicação de calcário. Os calcários calcíticos contêm, em média, $45 \%$ de $\mathrm{CaO}$, e os dolomíticos, em média, 20 a $40 \%$ de $\mathrm{CaO}$ e 10 a $20 \%$ de $\mathrm{MgO}$ (Malavolta, 1980). Em decorrência da baixa quantidade de $\mathrm{MgO}$ existente nos calcários calcíticos, o uso sistemático de tais corretivos afeta a relação Ca:Mg no solo (Fassbender \& Bornemisza, 1994).

Conforme Moore et al. (1961), o excesso de Ca em relação ao $\mathrm{Mg}$ na solução do solo prejudica a absorção deste último, assim como o excesso de $\mathrm{Mg}$ também prejudica a absorção de $\mathrm{Ca}$.

O presente trabalho teve como objetivo avaliar os efeitos da relação entre $\mathrm{Ca}$ e $\mathrm{Mg}$ do corretivo na nodulação, produção de matéria seca e concentra- ção de nutrientes na alfafa cultivada em Latossolo Vermelho-Escuro distrófico de cerrado.

\section{MATERIAL E MÉTODOS}

O experimento foi conduzido em condições de casa de vegetação do Departamento de Ciência do Solo da Universidade Federal de Lavras (UFLA), região sul de Minas Gerais, localizada nas coordenadas $21^{\circ} 14^{\prime} 6^{\prime \prime}$ de latitude sul e $45^{\circ} 00^{\prime}$ de longitude oeste, a uma altitude média de $900 \mathrm{~m}$.

O solo utilizado foi um Latossolo Vermelho-Escuro distrófico (Oxissolo) de cerrado $\left(\mathrm{pH}_{\text {água }}=4,8 ; \mathrm{P}_{\text {mehlich }}=\right.$ $2,0 \mathrm{mg} \mathrm{dm}^{-3} ; \mathrm{K}_{\text {Mehlich }}=40 \mathrm{mg} \mathrm{dm}^{-3} ; \mathrm{Ca}_{\mathrm{KCl}}=4,0 \mathrm{mmol}_{\mathrm{c}} \mathrm{dm}^{-3}$; $\mathrm{Mg}_{\mathrm{KCl}}=1,0 \mathrm{mmol}_{\mathrm{c}} \mathrm{dm}^{-3} ; \mathrm{S}=5,13 \mathrm{mg} \mathrm{dm}^{-3} ;$ M.O. $=$ $30,8 \mathrm{~g} \mathrm{~kg}^{-1} \mathrm{e} \mathrm{V}=11 \%$ ), coletado no município de Lavras, MG, na camada de $0-25 \mathrm{~cm}$ de profundidade. Após secagem, o solo foi homogeneizado e passado em peneira de malha de $4 \mathrm{~mm}$ para destorroamento e uniformização.

$\mathrm{O}$ delineamento experimental utilizado foi o inteiramente casualizado, em esquema fatorial 6 (tratamentos) x 6 (épocas de corte), com quatro repetições. Os tratamentos consistiram de diferentes relações $\mathrm{Ca}: \mathrm{Mg}$ do corretivo, aplicado na forma de $\mathrm{CaCO}_{3}+\mathrm{MgCO}_{3}$. Foram estudadas as relações $1: 0 ; 1: 1 ; 2: 1 ; 3: 1 ; 4: 1$ na dosagem de $3,9 \mathrm{t} \mathrm{ha}^{-1}$ do corretivo, e a relação $3: 1$ na dosagem de $7,8 \mathrm{t} \mathrm{ha}^{-1}$. A dose do corretivo foi calculada para elevar o índice de saturação por base, ao valor de $80 \%$ (exceto o último tratamento).

Dois meses após a aplicação dos tratamentos, o solo recebeu adubação básica nas seguintes dosagem em $\mathrm{mg} \mathrm{dm}{ }^{-3}$ : P - 200 (MAP), $\mathrm{K}-150(\mathrm{KCl}), \mathrm{S}-50\left(\mathrm{~K}_{2} \mathrm{SO}_{4}\right)$, B - 0,5 $\left(\mathrm{H}_{3} \mathrm{BO}_{3}\right), \mathrm{Co}-0,01\left(\mathrm{CoCl}_{2} .2 \mathrm{H}_{2} \mathrm{O}\right), \mathrm{Cu}-1,5$ $\left(\mathrm{CuSO}_{4} .5 \mathrm{H}_{2} \mathrm{O}\right)$, Mo - 0,1 $\left(\mathrm{H}_{2} \mathrm{MoO}_{4}\right), \mathrm{Mn}-3,5$ $\left(\mathrm{MnSO}_{4} \cdot \mathrm{H}_{2} \mathrm{O}\right)$ e $\mathrm{Zn}-5,0\left(\mathrm{ZnSO}_{4} \cdot 7 \mathrm{H}_{2} \mathrm{O}\right)$; essas doses estavam de acordo com Allen et al. (1976) e Malavolta (1980), no tocante a experimento em casa de vegetação; para o suprimento de $\mathrm{N}$ foi feita a inoculação das sementes com o rizóbio específico (Rhizobium meliloti).

Dez sementes escarificadas de alfafa, cultivar Crioula, foram semeadas em vasos de plástico com 5 litros de capacidade, e após o desbaste foram mantidas 5 plantas uniformes. Os vasos foram irrigados até que atingissem $70 \%$ do valor total de poros (VTP), e pesados diariamente a fim de se determinar a quantidade de água a ser reposta. O primeiro corte foi realizado três meses após a semeadura, e os posteriores foram feitos com base em $10 \%$ do florescimento total do experimento (em torno de 35 dias de intervalo entre cada corte). Após cada corte, o material foi levado à estufa $\left(65^{\circ} \mathrm{C}\right)$, para posterior obtenção do peso da matéria seca (MS). Foram determinados os teores de N, P, K, Ca, 
Mg e S da matéria seca da parte aérea, conforme Malavolta et al. (1997), e a proteína bruta foi obtida multiplicando-se o teor de $\mathrm{N}$ pelo fator 6,25. Após o último corte, as raízes foram lavadas em água desionizada/destilada, retirando-se todos os nódulos, para avaliação do peso fresco. Posteriormente, os nódulos foram colocados em placa-de-Petri e levados à estufa $\left(65^{\circ} \mathrm{C}\right)$ até atingir peso constante, e novamente submetidos à pesagem, para obtenção da massa seca dos nódulos.

Os resultados foram submetidos à análise de variância (teste F), de acordo com o delineamento proposto, e as médias dos tratamentos foram comparadas pelo teste de Tukey a 5\% de significância, conforme a metodologia descrita por Pimentel-Gomes (1990).

\section{RESULTADOS E DISCUSSÃO}

O peso da matéria seca da parte aérea, média de seis épocas de corte, é apresentado na Tabela 1. Verificou-se que a alfafa não respondeu significativamente às diferentes relações $\mathrm{Ca} \mathrm{e} \mathrm{Mg}$ do corretivo da acidez do solo. Tais dados confirmam os resultados obtidos por Hunter (1948) e Simsom et al. (1979), que, trabalhando com várias relações, encontraram respostas pouco significativas da alfafa a esse tipo

TABELA 1. Peso da matéria seca da parte aérea (média de seis cortes) e peso fresco e massa seca dos nódulos, em função dos tratamentos. Média de quatro repetições ${ }^{1}$.

\begin{tabular}{lccl}
\hline $\begin{array}{l}\text { Relação } \\
\text { Ca:Mg }\end{array}$ & $\begin{array}{c}\text { Matéria } \\
\text { seca }\end{array}$ & $\begin{array}{c}\text { Peso fresco } \\
\text { dos nódulos }\end{array}$ & $\begin{array}{l}\text { Massa seca } \\
\text { dos nódulos }\end{array}$ \\
\hline & $-----------\left(g /\right.$ vaso $\left.^{-1}\right)$ & ------------- \\
$1: 0$ & $10,3 \mathrm{~b}$ & $0,71 \mathrm{ab}$ & $0,12 \mathrm{abc}$ \\
$1: 1$ & $10,2 \mathrm{~b}$ & $0,34 \mathrm{~b}$ & $0,08 \mathrm{c}$ \\
$2: 1$ & $10,2 \mathrm{~b}$ & $0,91 \mathrm{ab}$ & $0,13 \mathrm{abc}$ \\
$3: 1$ & $11,2 \mathrm{~b}$ & $1,05 \mathrm{ab}$ & $0,22 \mathrm{ab}$ \\
$4: 1$ & $9,6 \mathrm{~b}$ & $1,43 \mathrm{a}$ & $0,25 \mathrm{a}$ \\
$3: 1$ & $15,2 \mathrm{a}$ & $0,40 \mathrm{~b}$ & $0,09 \mathrm{bc}$ \\
\hline Média & 11,1 & 0,81 & 0,15 \\
\hline D.M.S. & 1,8 & 0,92 & 0,13 \\
\hline
\end{tabular}

1 Médias seguidas por letras distintas diferem entre si a 5\% de probabilidade pelo teste de Tukey.

2 Nos cinco primeiros tratamentos foi aplicada uma dose equivalente a $3,9 \mathrm{t} \mathrm{ha}^{-1}$ de calcário e no último foi aplicada uma dose equivalente a $7,8 \mathrm{tha}^{-1}$. de tratamento. À aplicação do dobro da dosagem do corretivo (relação 3:1 - 7,8 t ha-1), a alfafa respondeu significativamente no aumento da produção de matéria seca. Resultados semelhantes foram obtidos por Kornelius \& Ritchey (1992), nas condições de cerrado, no qual observaram que a alfafa continua respondendo à aplicação de calcário, mesmo quando se aplica o dobro da recomendação indicada. Essa exigência de aplicação de calcário é confirmada por Rhykerd \& Overdahl (1972), ao relatarem que para produzir 10 toneladas de feno, a alfafa extrai do solo aproximadamente $350 \mathrm{~kg}$ de Ca. Freitas \& Pratt (1969) sugerem que para uma produção satisfatória dessa leguminosa seja necessário elevar o $\mathrm{pH}_{\text {(água) }}$ do solo ao redor de 6,0, o que foi confirmado pelos dados do presente trabalho (Tabelas 1 e 2).

Com relação às épocas de corte (Tabela 3 ), observou-se que as maiores produções de matéria seca foram obtidas no terceiro corte, confirmando os resultados encontrados por Jones \& Quagliato (1970), nas condições de Nova Odessa, SP, e por Moreira et al. (1997), nas condições de Lavras, MG.

Ao comparar os dados de peso fresco e massa seca dos nódulos (Tabela 1) com os de teor de $\mathrm{N}$ (Tabela 4), verificou-se que, exceto no tratamento $4: 1$, maior nodulação não resultou necessariamente num maior teor de $\mathrm{N}$ na matéria seca da parte aérea. Esse resultado corrobora as observações feitas por Bonner \& Varner (1976), que, trabalhando com soja, não encontraram correlações entre a massa seca de nódulos e o teor de $\mathrm{N}$ presente na matéria seca da parte aérea da planta. $\mathrm{O}$ teor médio de $\mathrm{N}$ foi de $32,0 \mathrm{~g} \mathrm{~kg}^{-1}$, o que corresponde a $20 \%$ de proteína bruta, estando este valor acima dos encontrados por Caldwell et al. (1969) e Moreira (1997), que obtiveram um teor médio de $27,0 \mathrm{~g} \mathrm{~kg}^{-1}$ de $\mathrm{N}$ na matéria seca, ou seja, em torno de $17 \%$ de proteína bruta.

As concentrações de $\mathrm{Ca}$ e $\mathrm{Mg}$ na parte aérea (Tabela 4) foram alteradas pelas diferentes relações de $\mathrm{Ca}: \mathrm{Mg}$ testadas, que proporcionaram acréscimo no teor de $\mathrm{Ca}$ e decréscimo no teor de $\mathrm{Mg}$, devido ao aumento da quantidade de Ca no corretivo. Em conseqüência disso, houve natural aumento no teor de Ca trocável no solo (Tabela 2), intensificando a competição catiônica desses dois elementos. Em todos os tratamentos, os teores de Ca na matéria seca da parte aérea ficaram na faixa de 10,0 a $20,0 \mathrm{~g} \mathrm{~kg}^{-1}$, con- 
siderada adequada em início de florescimento, conforme proposto por Pinkerton et al. (1997). No tratamento que recebeu o corretivo na relação $1: 1$, a menor quantidade de $\mathrm{Ca}$ existente no solo causou reduções significativas no processo de nodulação (Tabela 1). Conforme Munns (1978), o Ca tem grande importância no processo de nodulação. A carência desse elemento produz efeitos adversos nas propriedades fisiológicas das plantas e na multiplicação das bactérias que formam os nódulos, as quais sofrem distúrbios na integridade estrutural da parede celular (Andrew \& Johnson, 1976).

Nos tratamentos 1:0 e 1:1 (Tabela 4), os teores de $\mathrm{Mg}$ ficaram abaixo e acima, respectivamente, do nível crítico de 3,5 $\mathrm{g} \mathrm{kg}^{-1}$ proposto por Martins et al. (1990), porém os demais tratamentos alcançaram um nível de suficiência adequado. Tal fato ocorreu porque a quantidade de Mg no solo (Tabela 2), no tratamento 1:0, foi, provavelmente, insuficiente para manter um teor adequado na planta, enquanto no tratamento 1:1, embora a quantidade de $\mathrm{Mg}$ no solo te- nha ficado num nível considerado adequado por Raij (1991), que é igual ou superior a $8,0 \mathrm{mmol}_{\mathrm{c}} \mathrm{dm}^{-3}$; a alfafa absorveu uma quantidade maior que suas necessidades, proporcionando um aumento da concentração na matéria seca. Observando-se as Tabelas 1 e 2, nota-se que o teor original de $\mathrm{Mg}$ no solo $\left(1,0 \mathrm{mmol}_{\mathrm{c}} \mathrm{dm}^{-3}\right)$ foi, aparentemente, suficiente para ocasionar uma boa nodulação. $\mathrm{O}$ aumento nas doses de $\mathrm{Mg}$ no corretivo promoveram decréscimo no peso seco dos nódulos.

Conquanto não apresentassem diferença significativa (Tabela 4), os teores de P na matéria seca da parte aérea mantiveram-se entre 2,3 e $2,5 \mathrm{~g} \mathrm{~kg}^{-1}$. Valores que podem ser julgados satisfatórios, considerando-se que o nível crítico para alfafa, estabelecido por Andrew \& Robins (1969) e Moreira (1997), é de $2,4 \pm 0,1 \mathrm{~g} \mathrm{~kg}^{-1}$.

Os teores de K na parte aérea, nos diversos tratamentos (Tabela 4), situaram-se abaixo dos valores considerados adequados por Pinkerton et al. (1997), que é de 20,0 a 35,0 $\mathrm{g} \mathrm{kg}^{-1} \mathrm{em}$ início de florescimento.

TABELA 2. Resultados das análises químicas do solo antes do plantio e depois do último corte. Média de quatro repetições.

\begin{tabular}{|c|c|c|c|c|c|c|c|}
\hline Relação Ca:Mg ${ }^{1}$ & $\begin{array}{c}\mathrm{pH} \\
\text { em } \mathrm{H}_{2} \mathrm{O}\end{array}$ & $\begin{array}{c}\mathrm{P} \\
\left(\mathrm{mg} \mathrm{dm}^{-3}\right)\end{array}$ & $\begin{array}{c}\mathrm{K} \\
\left(\mathrm{mg} \mathrm{dm}^{-3}\right)\end{array}$ & $\begin{array}{c}\mathrm{Ca} \\
\left(\mathrm{mmol}_{\mathrm{c}} \mathrm{dm}^{-3}\right)\end{array}$ & $\begin{array}{c}\mathrm{Mg} \\
\left(\mathrm{mmol}_{\mathrm{c}} \mathrm{dm}^{-3}\right)\end{array}$ & $\begin{array}{c}\mathrm{S} \\
\left(\mathrm{mg} \mathrm{dm}^{-3}\right)\end{array}$ & $\begin{array}{c}\mathrm{Al} \\
\left(\mathrm{mmol}_{\mathrm{c}} \mathrm{dm}^{-3}\right)\end{array}$ \\
\hline $1: 0$ & 6,4 & 50,3 & 99,3 & 33,0 & 1,0 & 15.2 & 1.0 \\
\hline $1: 1$ & 6,3 & 48,0 & 84,7 & 22,0 & 20,0 & 14,7 & 1,0 \\
\hline $2: 1$ & 6,0 & 42,5 & 89,3 & 19,0 & 11,0 & 15,3 & 1,0 \\
\hline $3: 1$ & 6,3 & 53,3 & 80,7 & 29,0 & 12,0 & 14,7 & 1,0 \\
\hline $4: 1$ & 6,1 & 62,8 & 94,0 & 32,0 & 11,0 & 14,5 & 1,0 \\
\hline $3: 1$ & 6,6 & 69,0 & 64,0 & 35,0 & 17,0 & 15,0 & 1,0 \\
\hline Média & 6,3 & 54,3 & 85,3 & 28,3 & 12,0 & 14,9 & 1,0 \\
\hline 1:0 & 4,8 & 14,0 & 66,0 & 18,0 & 1,0 & 4,9 & 1,0 \\
\hline $1: 1$ & 4,9 & 12,0 & 65,3 & 13,0 & 8,0 & 4,8 & 2,0 \\
\hline $2: 1$ & 4,8 & 14,0 & 64,3 & 16,0 & 6,0 & 4,9 & 1,0 \\
\hline $3: 1$ & 4,7 & 9,5 & 57,0 & 16,0 & 3,0 & 6,2 & 2,0 \\
\hline $4: 1$ & 4,9 & 10,0 & 55,5 & 17,0 & 4,0 & 4,9 & 2,0 \\
\hline $3: 1$ & 5,8 & 8,0 & 44,3 & 30,0 & 9,0 & 6,2 & 1,0 \\
\hline Média & 5,0 & 11,3 & 58,7 & 18,3 & 5,2 & 5,3 & 1,5 \\
\hline Testemunha $^{2}$ & 4,8 & 2,0 & 40,0 & 4,0 & 1,0 & 5,1 & 6,0 \\
\hline
\end{tabular}

${ }_{1}^{1}$ Nos cinco primeiros tratamentos foi aplicada uma dose equivalente a $3,9 \mathrm{t} \mathrm{ha}^{-1}$ de calcário e no último foi aplicada uma dose equivalente a $7,8 \mathrm{t}$ ha ${ }^{-1}$.

2 Análise química do solo antes da aplicação dos tratamentos. 
TABELA 3. Influência da época de corte na produção de matéria seca da parte aérea $\left(\mathrm{g} \mathrm{vaso}^{-1}\right)^{1}$.

\begin{tabular}{lrrrrrr}
\hline \multirow{2}{*}{$\begin{array}{l}\text { Relação } \\
\text { Ca:Mg }\end{array}$} & \multicolumn{7}{c}{ Época de corte } \\
\cline { 2 - 7 } & 1 & 2 & 3 & 4 & 5 & 6 \\
\hline $1: 0$ & 8,8 & 13,0 & 16,1 & 9,7 & 7,4 & 6,8 \\
$1: 1$ & 11,0 & 13,8 & 15,2 & 8,7 & 6,3 & 6,3 \\
$2: 1$ & 9,2 & 14,6 & 15,0 & 8,0 & 7,1 & 7,5 \\
$3: 1$ & 10,3 & 14,6 & 16,0 & 9,4 & 8,1 & 8,8 \\
$4: 1$ & 8,3 & 14,2 & 13,7 & 8,0 & 6,0 & 7,5 \\
$3: 1$ & 14,0 & 18,1 & 16,1 & 17,6 & 14,6 & 11,5 \\
\hline Média & $10,3 \mathrm{~b}$ & $14,7 \mathrm{a}$ & $15,3 \mathrm{a}$ & $10,5 \mathrm{~b}$ & $8,3 \mathrm{c}$ & $8,1 \mathrm{c}$ \\
\hline D.M.S. & 1,8 & & & & & \\
\hline
\end{tabular}

${ }^{1}$ Médias seguidas por letras distintas diferem entre si a $5 \%$ de probabilidade pelo teste de Tukey.

2 Nos cinco primeiros tratamentos foi aplicada uma dose equivalente a $3,9 \mathrm{tha}^{-1}$ de calcário e no último foi aplicada uma dose equivalente a $7,8 \mathrm{tha}^{-1}$.

TABELA 4. Teores de N, P, K, Ca, Mg e S na matéria seca da parte aérea, média dos seis cortes, em função dos tratamentos. Média de quatro repetições ${ }^{1}$.

\begin{tabular}{lllllll}
\hline $\begin{array}{l}\text { Relação } \\
\text { Ca:Mg }\end{array}$ & $\mathrm{N}$ & $\mathrm{P}$ & $\mathrm{K}$ & $\mathrm{Ca}$ & $\mathrm{Mg}$ & $\mathrm{S}$ \\
\hline & & & & & & \\
\hline $1: 0$ & $32,9 \mathrm{ab}$ & $2,4 \mathrm{a}$ & $16,4 \mathrm{~b}$ & $19,4 \mathrm{a}$ & $1,5 \mathrm{~d}$ & $1,1 \mathrm{c}$ \\
$1: 1$ & $30,0 \mathrm{~b}$ & $2,3 \mathrm{a}$ & $17,3 \mathrm{ab}$ & $12,3 \mathrm{~b}$ & $5,5 \mathrm{a}$ & $1,4 \mathrm{~b}$ \\
$2: 1$ & $31,4 \mathrm{ab}$ & $2,5 \mathrm{a}$ & $17,2 \mathrm{~b}$ & $14,4 \mathrm{~b}$ & $4,5 \mathrm{~b}$ & $1,5 \mathrm{ab}$ \\
$3: 1$ & $30,9 \mathrm{~b}$ & $2,3 \mathrm{a}$ & $17,1 \mathrm{~b}$ & $17,9 \mathrm{a}$ & $4,0 \mathrm{c}$ & $1,4 \mathrm{~b}$ \\
$4: 1$ & $34,6 \mathrm{a}$ & $2,3 \mathrm{a}$ & $18,5 \mathrm{a}$ & $17,6 \mathrm{a}$ & $3,6 \mathrm{~d}$ & $1,6 \mathrm{ab}$ \\
$3: 1$ & $32,0 \mathrm{ab}$ & $2,4 \mathrm{a}$ & $15,0 \mathrm{c}$ & $18,9 \mathrm{a}$ & $4,5 \mathrm{~b}$ & $1,7 \mathrm{a}$ \\
\hline Média & 32,0 & 2,4 & 16,9 & 16,8 & 3,9 & 1,5 \\
\hline D.M.S. & 3,7 & 0,3 & 1,3 & 2,1 & 0,4 & 0,2 \\
\hline
\end{tabular}

${ }^{1}$ Médias seguidas por letras distintas diferem entre si a $5 \%$ de probabilidade pelo teste de Tukey.

2 Nos cinco primeiros tratamentos foi aplicada uma dose equivalente a $3,9 \mathrm{tha}^{-1}$ de calcário e no último foi aplicada uma dose equivalente a 7,8 tha $^{-1}$.

Mesmo apresentando alta quantidade de $\mathrm{K}$ no solo (Tabela 2), a planta não conseguiu manter um teor adequado na matéria seca da parte aérea, o que se deve, provavelmente, ao efeito da inibição competitiva entre o $\mathrm{Ca}$ e $\mathrm{K}$ e $\mathrm{Mg}$ e $\mathrm{K}$, tal como reportado por Marschner (1995) e Malavolta et al. (1997).

Os teores de S na matéria seca da parte aérea (Tabela 4) apresentaram-se sempre aquém do nível crítico de 2,0 a 2,2 $\mathrm{g} \mathrm{kg}^{-1}$ apontado por Lanyon \& Griffith (1988) e de 2,5 a 4,0 $\mathrm{g} \mathrm{kg}^{-1}$ estabelecido por Pinkerton et al. (1997), porém acima dos obtidos por Moreira et al. (1997), que ao trabalharem nas mesmas condições edafoclimáticas, encontraram um teor médio de $1,4 \mathrm{~g} \mathrm{~kg}^{-1}$ na MS da parte aérea. A relação N/S na planta, que pode ser considerada um índice confiável na avaliação do estado nutricional, mostrou, no presente estudo, valor médio de 19,29, sendo semelhante ao de outros trabalhos citados por Lanyon \& Griffith (1988). Embora sem significância estatística, houve um aumento no valor de $\mathrm{S}$ no tratamento com o dobro da dose recomendada. Malavolta (1980) menciona que cátions acompanhantes como o $\mathrm{Ca}$ e $\mathrm{Mg}$ aumentam a absorção do sulfato, forma predominantemente absorvida pelas raízes. Martini \& Mutters (1984) observaram que altas doses de $\mathrm{CaCO}_{3}$ reduzem significativamente a disponibilidade de $\mathrm{S}$, o que também foi observado no tratamento 1:0 (Tabela 4).

Houve uma acidificação natural do solo (Tabela 2). O pH variou antes do plantio, de 6,0 no tratamento 2:1 a 6,6 no tratamento com o dobro da dosagem recomendada, e após o último corte variou de 4,7 no tratamento $3: 1$ a 5,8 no tratamento com o dobro da dosagem recomendada. Tal resultado, possivelmente, ocorreu em decorrência da dissociação do $\mathrm{CO}_{2}$ liberado através da respiração radicular, pelo íon sulfato presente no $\mathrm{K}_{2} \mathrm{SO}_{4}$, ou pela mineralização dos resíduos vegetais (Malavolta, 1980; Raij, 1991; Tisdale et al., 1993).

\section{CONCLUSÕES}

1. Em Latossolo Vermelho-Escuro distrófico, mudanças na relação Ca:Mg no corretivo de acidez do solo não afetam a produção de matéria seca.

2. A relação 4:1 apresenta maior eficiência na nodulação da alfafa

3. $\mathrm{O}$ excesso de $\mathrm{Ca}$ ou de $\mathrm{Mg}$ acarreta uma inibição competitiva com o K na alfafa.

\section{REFERÊNCIAS}

ALLEN, S.E.; TERMAN, G.L.; CLEMENTS, L.B. Greenhouse techniques for soil-plant-fertilizer research. Muscle Shoals: National Fertilizer Development Center, 1976. 55p.

ANDREW, C.S.; JOHNSON, A.D. Effect of calcium, pH and nitrogen on the growth and chemical composition 
of some tropical and temperate pasture legumes. II. Chemical composition (calcium, nitrogen, potassium, magnesium, sodium and phosphorus). Australian Journal of Agricultural Research, Melbourne, v.27, n.2, p.625-636, 1976.

ANDREW, C.S.; ROBINS, M.F. The effect of phosphorus on the growth and chemical composition of some tropical pasture legumes. I. Growth and critical percentages of phosphorus. Australian Journal of Agricultural Research, Melbourne, v.20, n.2, p.665-674, 1969.

ANUÁRIO ESTATÍSTICO DO BRASIL: 1987/1988. Rio de Janeiro: IBGE, n.48, p.331, 1989. 715p.

BONNER, J.; VARNER, J.E. Plant biochemistry. New York: Academic Press, 1976. 925p.

CALDWELL, A.C.; SEIM, E.C.; REHM, G.W. Sulfur effects on the elemental composition of alfalfa (Medicago sativa L.) and corn (Zea mays L.). Agronomy Journal, Madison, v.61, n.2, p.632-634, 1969.

FASSBENDER, H.W.; BORNEMISZA, E. Química del suelos con énfases en suelos de América Latina. San José: IICA, 1994. 420p.

FREITAS, L.M.M.; PRATT, P.F. Resposta de três leguminosas a calcário em diversos solos ácidos de São Paulo. Pesquisa Agropecuária Brasileira, Série Agronomia, Rio de Janeiro, v.4, n.2, p.89-95, 1969.

HUNTER, A.S. Yield and composition of alfalfa as affected by variations in the calcium-magnesium ratio in the soil. Soil Science, Baltimore, v.67, n.1, p.53-62, 1948.

JONES, M.B.; QUAGLIATO, J.L. Respostas de quatro leguminosas tropicais e da alfafa a vários níveis de enxofre. Pesquisa Agropecuária Brasileira, Série Agronomia, Rio de Janeiro, v.5, n.3, p.359-363, 1970 .

KORNELIUS, E.; RITCHEY, K.D. Comportamento da alfafa em diferentes níveis de acidez do solo. Pesquisa Agropecuária Brasileira, Brasília, v.27, n.2, p.241-246, 1992.

LANYON, L.E.; GRIFFITH, W.K. Nutrition and fertilizer use. In: HANSON, A.A.; BARNES, D.K.; HILL, R.R. (Eds.). Alfalfa and alfalfa improvement. Madison: SSSA, 1988. p.333-364.

MALAVOLTA, E. Elementos da nutrição mineral de plantas. São Paulo: Agronômica Ceres, 1980. 251p.
MALAVOLTA, E.; VITTI, G.C.; OLIVEIRA, S.A. Avaliação do estado nutricional das plantas; princípios e aplicações. Piracicaba: POTAFOS, 1997.319p.

MARSCHNER, H. Mineral nutrition of higher plants. London: Academic Press, 1995. 889p.

MARTINI, J.A.; MUTTERS, R.G. Effects of liming and fertilization on sulfur availability, mobility and uptake in cultivated soils of South Carolina. Soil Science, Baltimore, v.138, n.6, p.403-410, 1984.

MARTINS, C.E.; CÓSER, A.C.; OLIVEIRA, F.T.T.; SARAIVA, O.F. Avaliação dos nutrientes limitantes ao crescimento de alfafa em solo aluvial. Revista da Sociedade Brasileira de Zootecnia, Viçosa, v.19, n.4, p.333-339, 1990 .

MOORE, D.P.; OVERSTREET, R.; JACOBSON, L. Uptake of magnesium and its interactions with calcium in excised barley roots. Plant Physiology, Rockville, v.36, n.3, p.290-295, 1961.

MOREIRA, A. Efeitos de fontes e doses de fósforo na alfafa (Medicago sativa L.) e centrosema (Centrosema pubescens Benth.) e avaliação de extratores. Piracicaba: USP-ESALQ, 1997. 107p. Tese de Mestrado.

MOREIRA, A.; CARVALHO, J.G.; EVANGELISTA, A.R. Efeito de doses de enxofre na produção e composição mineral da alfafa. Pesquisa Agropecuária Brasileira, Brasília, v.32, n.5, p.533-538, 1997.

MUNNS, D.N. Soil acidity and nodulation. In: ANDREW, C.S.; KAMPRATH, E.J. (Eds.). Mineral nutrition of legumes in tropical and subtropical soils. Melbourne: CSIRO, 1978. p.247-264.

NUERNBERG, N.J. Técnicas de produção de alfafa. In: PEIXOTO, A.M.; MOURA, J.C.; FARIA, V.P. (Eds.). CONGRESSO BRASILEIRO DE PASTAGENS, 8., 1986. Piracicaba. Anais... Piracicaba: FEALQ, 1986. p.145-160.

NUERNBERG, N.J.; MILAN, P.A.; SILVEIRA, C.A.A. Manual de produção de alfafa. Florianópolis: EMPASC, 1990. 102p.

PIMENTEL-GOMES, F. Curso de Estatística Experimental. Piracicaba: Nobel, 1990. 468p.

PINKERTON, A.; SMITH, F.W.; LEWIS, D.C. Pasture Species. In: REUTER, D.J.; ROBINSON, J.B. (Eds.). Plant analysis; an interpretation manual. Collingwood: CSIRO, 1997. p.285-343. 
RAIJ, B. van. Fertilidade do solo e adubação. São Paulo: Ceres/Piracicaba: POTAFOS, 1991. 343p.

RHYKERD, C.L.; OVERDAHL, C.J. Nutrition and fertilizer use. In: HANSON, C.H. (Ed.). Alfalfa Science and Technology. Madison: SSSA, 1972. p.437-468.

SILVA, R. Efeito de níveis de correção em diferentes relações Ca:Mg sobre o comportamento das variedades UFV 1 e IAC 2 da soja em solos de cerra- do. Viçosa: UFV, 1981. 35p. Dissertação de Mestrado.

SIMSOM, C.R.; COREY, R.B.; SUMMER, M.E. Effect of varying $\mathrm{Ca}: \mathrm{Mg}$ ratios on yield and composition of corn (Zea mays) and alfalfa (Medicago sativa). Communications in Soil Science and Plant Analysis, New York, v.10, n.1/2, p.153-162, 1979.

TISDALE, S.L.; NELSON, W.L.; BEATON, J.D.; HAVLIN, J.L. Soil fertility and fertilizers. New York: Macmillan, 1993. 634p. 